\title{
RoseMerry: A Baseline Message-level Sentiment Classification System
}

\author{
Huizhi Liang, Richard Fothergill and Timothy Baldwin \\ The University of Melbourne \\ VIC 3010, Melbourne \\ oklianghuizi@gmail.com, r.fothergill@student.unimelb.edu.au, tb@ldwin.net
}

\begin{abstract}
In this paper, we propose a baseline messagelevel sentiment classification method, as developed for SemEval-2015 Task 10, Subtask B. This system leverages both hand-crafted features and message-level embedding features, and uses an SVM classifier for messagelevel sentiment classification. In pre-training the embedding features, we use one million randomly-selected tweets. We present results over SemEval-2015 Task 10, Subtask B, as well as the Stanford Sentiment Treebank. Our experiments show the effectiveness of our method over both datasets.
\end{abstract}

\section{Introduction}

The rise of social media such as blogs and microblogs (e.g., Twitter) has fueled interest in sentiment analysis (Liu, 2012; Pang and Lee, 2008). One of the most popular settings for carrying out sentiment analysis is at the sentence level or over individual micro-blog posts, using the simple threelabel class set of POSITIVE, NEGATIVE and NEUTRAL (Liu, 2012; Pang and Lee, 2008; Rosenthal et al., 2014). Sentiment classification has been shown to have utility in various business intelligence applications, including product marketing, identifying new business opportunities, and managing a company's reputation (Liu, 2012; Pang and Lee, 2008).

Learning effective features plays an important role in building sentiment classification systems (Liu, 2012; Pang and Lee, 2008). For example, the winning system in the SemEval-2013 message polarity classification task (Nakov et al.,
2013) was based on a rich set of hand-tuned features such as word-sentiment association lexicon features, word $n$-grams, punctuation, and emoticons, which were combined using a simple SVM-based classifier (Mohammad et al., 2013). Recently, there has been a surge of interest in representation learning - automatically learning word and document representations, often in the form of continuous-valued vectors or "embeddings" - using auto-encoders or neural network language models (Mikolov et al., 2013; Le and Mikolov, 2014). Of particular relevance to message-level sentiment analysis, Tang et al. (2014) proposed a deep learning approach to learn sentiment-specific word representation features, and Le and Mikolov (2014) proposed a neural network auto-encoder to learn message-level vectors.

In this paper, we detail RoseMerry, a (strong) baseline sentiment analysis method that combines hand-crafted features with message-level ${ }^{1}$ embeddings generated by doc2vec (Le and Mikolov, 2014), using a linear-kernel SVM.

\section{The Proposed Method}

The proposed method combines a set of handcrafted features with automatically-generated message-level representation features. The features are concatenated into a combined feature representation, and fed into a linear-kernel SVM learner using LibSVM (Chang and Lin, 2011). The

\footnotetext{
${ }^{1}$ Throughout the paper, we will use "message" as a generic term to refer to both tweets and also sentences in the case of the Stanford Sentiment Treebank. Note that the method could potentially be applied to any granularity of document.
} 


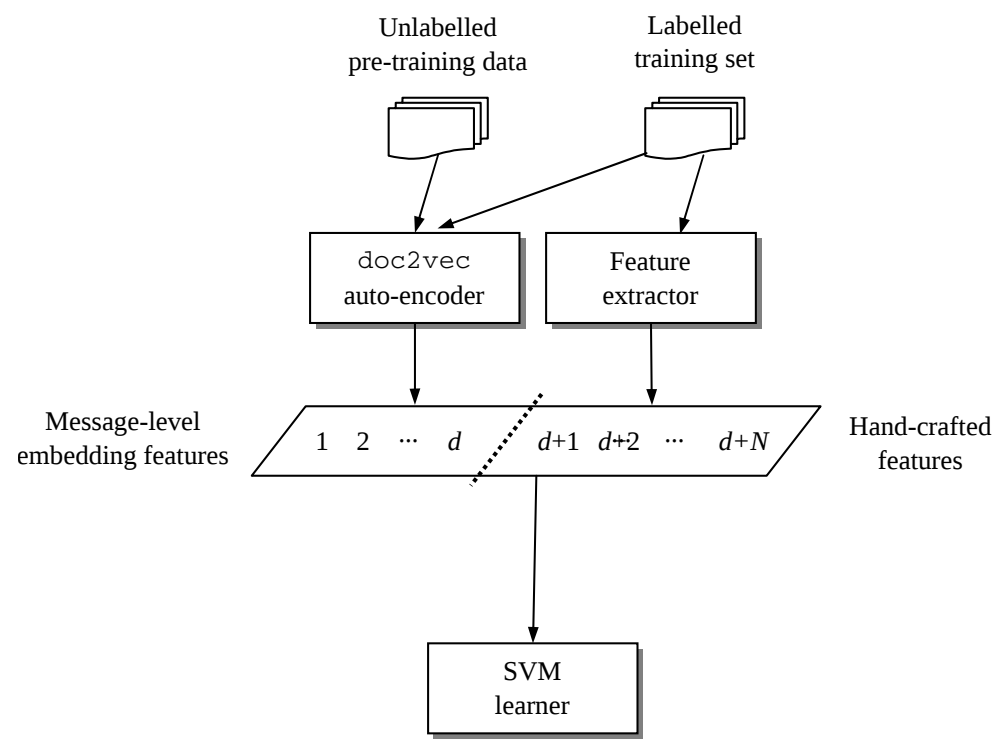

Figure 1: System architecture

architecture of the method is shown in Figure 1.

Our interest in sentiment analysis stems from a desire to use it as part of a commercial text analytics system. As such, there is an overarching constraint associated with the system and all third-party components must be licensed in a manner which is compatible with commercial use. In our description below, we point out places where we were unable to use notable resources because of this constraint.

The message-level embeddings are pre-trained using doc 2 vec over the combination of the training data and a random sample of $1 \mathrm{M}$ English tweets, as detailed in Section 2.1. The hand-crafted features are based heavily off the work of Mohammad et al. (2013), and are detailed in Section 2.2. Finally, the $d$-dimensional message-level embedding is concatenated with the $N$-dimensional hand-crafted features to form a $d+N$-dimensional combined feature vector. We experiment with each of the two feature subsets, in addition to the combined feature set.

One significant divergence from Mohammad et al. (2013) is that we do not use many of the sentiment lexicons, due to non-commercial licensing. Given that one of the key findings in that work was that lexicons are one of the most reliable features, we expect that this will have a large impact on our results.

\subsection{Message-level embeddings}

The message-level embeddings are generated using doc2vec (Le and Mikolov, 2014). In this framework, words and documents are represented in a common $d$-dimensional space, using real-valued vectors. The embeddings are learned by prediction of each word in a given document based on the document embedding and word embeddings of its surrounding context. The document vector acts as another word which captures the larger context of a word that is missing from its immediate word context.

The word and document vectors are trained using stochastic gradient descent, based on back propagation.

After pre-training, the document vector of each training document is used as its representation, and test documents are fed through the pre-trained autoencoder to generate a message-level embedding.

\subsection{Hand-crafted features}

The hand-crafted features are largely lexical:

- word $n$-grams: binary features capturing the presence or absence of word $n$-grams observed in the training data, i.e. contiguous sequences of $n$ words ( $n \in\{1,2,3,4\}$ ); we also included binary features for non-contiguous 3 - and 4grams included in the training data ( $n$-grams 
with one non-final word removed)

- character $n$-grams: continuous features capturing the proportion of contiguous character $n$ grams $(n \in\{3,4,5\})$ of each type observed in the training data, which make up a given message

- proportion of words in all caps: the proportion of words which are in all caps (e.g. YAY)

- punctuation features: the proportion of tokens which are made up of multiple exclamation marks, question marks, or a combination of the two (e.g. ??!)

- elongated words: the proportion of words which have "elongated" vowels, i.e. a given vowel repeated more than twice (e.g. coool)

- proportion of emoticons: the proportion of tokens which are (a) positive- and (b) negativepolarity emoticons, as identified by Chris Potts' scripts $^{2}$

- polarity of message-final emoticon: if the last token is a polarised emoticon, its polarity (NEGATIVE, POSITIVE or None)

- negated words: the presence or absence of words in "negated contexts", where a negated context is defined as span from a negation word $^{3}$ to a punctuation mark (matching the regular expression $[, .: ;$ ! ? ])

\section{Experiments}

In this section, we will detail the experimental setup and the results of our experiments.

\subsection{Datasets}

We evaluate our method over two labelled datasets, and also two unlabelled datasets to pre-train doc 2 vec, as detailed below.

\footnotetext{
${ }^{2}$ http://sentiment. christopherpotts.net/ tokenizing.html

${ }^{3}$ Defined based on Chris Potts' word list: http:// sentiment. christopherpotts. net/lingstruc. html.
}

\begin{tabular}{lccc}
\hline & $\begin{array}{c}\text { Training } \\
\text { Set }\end{array}$ & $\begin{array}{c}\text { Development } \\
\text { Set }\end{array}$ & $\begin{array}{c}\text { Test } \\
\text { Set }\end{array}$ \\
\hline POSITIVE & 3043 & 438 & 1038 \\
NEGATIVE & 1177 & 212 & 365 \\
NEUTRAL & 4082 & 542 & 987 \\
\hline
\end{tabular}

Table 1: The number of POSITIVE, NEGATIVE, NEUTRAL documents in the SemEval-2015 dataset

\begin{tabular}{lcc}
\hline & Training Set & Test Set \\
\hline POSITIVE & 3606 & 444 \\
NEGATIVE & 3304 & 428 \\
NEUTRAL & 1623 & 226 \\
\hline
\end{tabular}

Table 2: The number of POSITIVE, NEGATIVE and NEUTRAL sentences in the Stanford Sentiment Treebank dataset

\subsubsection{Labelled Datasets}

SemEval-2015 Dataset: the official SemEval2015 Task 10, subtask B dataset, comprised of tweets which have been hand-labelled for sentiment at the message-level (in terms of POSITIVE, NEGATIVE and NEUTRAL sentiment). The dataset is partitioned into three components, as detailed in Table $1:^{4}$ (1) training set, (2) development set, and (3) test set.

Stanford Sentiment Treebank Dataset: a collection of movie review documents from wwW. rottentomatoes.com, which have been sentence tokenised and annotated for sentiment at the sentence level (Maas et al., 2011) and prepartitioned into training and test data, as detailed in Table 2. Socher et al. (2013) additionally annotated the data at the phrase and lexical levels, but we use only the sentence-level annotations in this paper.

\subsubsection{Unlabelled Datasets}

Twitter Dataset: a random sample of $10 \mathrm{M}$ English tweets from a 5.3TB Twitter dataset crawled from 18 June to 4 Dec, 2014 using the Twitter Trending API. This is used as additional data to pre-train the message-level embeddings for the SemEval2015 Dataset.

IMDB Dataset: a $100 \mathrm{~K}$ sentence movie review dataset from www.imdb.com, collected by Maas

\footnotetext{
${ }^{4}$ As the labels have not been released for the progress test set, we omit this from the table.
} 
et al. (2011). This is used as additional data to pretrain the message-level embeddings for the Stanford Sentiment Treebank dataset.

\subsection{Experimental setup}

To evaluate the effectiveness of the different feature sets, we report on results as follows:

- RM-manual: only hand-crafted features

- RM-doc2vec: only message-level embeddings

- RM-all: both hand-crafted features and message-level embeddings

As our primary evaluation metric, we use $\mathrm{F} 1_{\mathrm{PN}}$, which is the average $F 1_{P N}$ for the POSITIVE (i.e., $F 1_{\text {pos }}$ ) and NEGATIVE classes (i.e., $F 1_{\text {neg }}$ ):

$$
\mathrm{F} 1_{\mathrm{PN}}=\frac{\mathrm{F} 1_{\text {pos }}+\mathrm{F} 1_{\text {neg }}}{2}
$$

We also report the overall classification accuracy (Acc) across the three classes, and the $\mathrm{F} 1_{\mathrm{PN}}$ score of each class (i.e., $F 1_{\text {pos }}, F 1_{\text {neg }}$ and $F 1_{\text {neu }}$ ).

For the message-level embeddings, we used $d=$ 100 and a context window size of 10 . We used LibSVM with a linear-kernel and default parameter settings.

\subsection{Experimental results}

In this section, we present the results first over the SemEval-2015 datasets, and then over the Stanford Sentiment Treebank.

\subsubsection{Results for SemEval-2015}

The results for the SemEval-2015 test set and progress test set are shown in Table 3. Figure 2a is a learning curve of RM-doc2vec, pre-trained over varying numbers of documents. We can see that the results plateau at $1 \mathrm{M}$ tweets; this is the document collection size we used for pre-training RM-doc2vec and RM-all in our official runs. The overall Acc and F1 of each class for the three feature sets are shown in Figure 2b. RM-doc2vec is marginally better than RM-manual overall, and for the NEGATIVE class in particular. When combined, RM-all outperforms the two component feature sets across all classes, pointing to (weak) complementarity between the two feature sets.

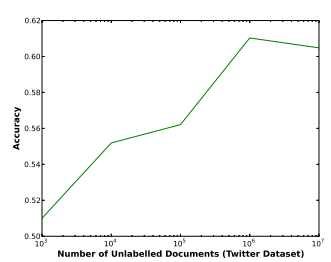

(a) Learning curve

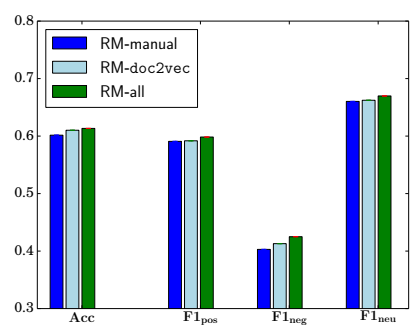

(b) Acc and per-class F1 score
Figure 2: The learning curve for RM-doc2vec, and the Acc, $F 1_{\text {pos }}, F 1_{\text {neg }}$, and $F 1_{\text {neu }}$ results for SemEval-2015

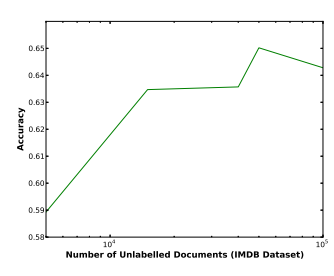

(a) Learning curve

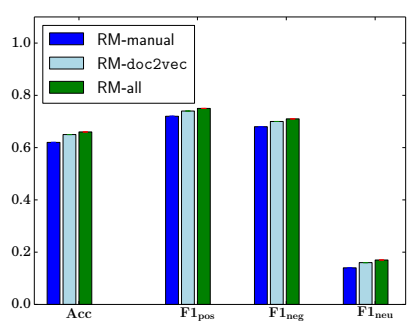

(b) Acc and per-class F1 score
Figure 3: The learning curve for RM-doc2vec, and the Acc, $F 1_{\text {pos }}, F 1_{\text {neg }}$, and $F 1_{\text {neu }}$ results for the Stanford Sentiment Treebank

\subsubsection{Results for the Stanford Sentiment Treebank}

The learning curve for RM-doc2vec over the Stanford Sentiment Treebank with varying numbers of unlabelled (IMDB) documents is given in Figure 3a. RM-doc2vec performed best when pretrained over $50 \mathrm{~K}$ documents (plus the Stanford Sentiment Treebank data), and this is the model we include in the remainder of our results over this dataset. Figure $3 \mathrm{~b}$ shows the Acc, in addition to the per-class F1 over the Stanford Sentiment Treebank for the three feature sets. The overall trend is strikingly similar to that for SemEval-2015, with the combined feature set performing marginally better than the two component feature sets in all cases.

\section{Conclusion}

In this paper, we described the method used in our official submission to the SemEval-2015 message polarity classification task, which combines message-level embeddings with hand-crafted features using a simple linear-kernel SVM. We pre- 


\begin{tabular}{cc||ccccc}
\hline \multicolumn{2}{c}{ Test Set } & \multicolumn{5}{c}{ Progress Test Set } \\
\hline Twitter & Twitter & LiveJournal & SMS & Twitter & Twitter & Twitter \\
2015 & 2015 Sarcasm & 2014 & 2013 & 2013 & 2014 & 2014 Sarcasm \\
\hline 0.5118 & 0.4962 & 0.6254 & 0.5300 & 0.5233 & 0.6127 & 0.4925 \\
\hline
\end{tabular}

Table 3: The official evaluation results for the SemEval-2015 Test and Progress Test set $\left(\mathrm{F} 1_{\mathrm{PN}}\right)$

sented results over the SemEval-2015 dataset and Stanford Sentiment Treebank, and showed that the combined feature achieved the best results. The difference between the combined feature set and the two component feature sets is not statistically significant (based on randomised estimation, $p>0.05$ ). While we were not able to achieve state-of-the-art results, we commend the proposed approach as a strong baseline method.

\section{Acknowledgements}

This research was supported by the Australian Research Council. The authors would like to acknowledge the support of Pitchwise (www.pitchwi. se), and also the coding assistance of Fei Liu.

\section{References}

Chih-Chung Chang and Chih-Jen Lin. 2011. LIBSVM: A library for support vector machines. ACM Transactions on Intelligent Systems and Technology, 2:27:127:27.

Quoc V. Le and Tomas Mikolov. 2014. Distributed representations of sentences and documents. CoRR, abs/1405.4053.

Bing Liu. 2012. Sentiment Analysis and Opinion Mining. Synthesis Lectures on Human Language Technologies. Morgan \& Claypool Publishers.

Andrew L. Maas, Raymond E. Daly, Peter T. Pham, Dan Huang, Andrew Y. Ng, and Christopher Potts. 2011. Learning word vectors for sentiment analysis. In Proceedings of the 49th Annual Meeting of the Association for Computational Linguistics: Human Language Technologies, pages 142-150, USA.

Tomas Mikolov, Kai Chen, Greg Corrado, and Jeffrey Dean. 2013. Efficient estimation of word representations in vector space. CoRR, abs/1301.3781.

Saif M. Mohammad, Svetlana Kiritchenko, and Xiaodan Zhu. 2013. NRC-Canada: Building the stateof-the-art in sentiment analysis of tweets. CoRR, abs/1308.6242.

Preslav Nakov, Sara Rosenthal, Zornitsa Kozareva, Veselin Stoyanov, Alan Ritter, and Theresa Wilson.
2013. Semeval-2013 task 2: Sentiment analysis in Twitter. In Second Joint Conference on Lexical and Computational Semantics (*SEM), Volume 2: Proceedings of the Seventh International Workshop on Semantic Evaluation (SemEval 2013), pages 312-320, USA.

Bo Pang and Lillian Lee. 2008. Opinion mining and sentiment analysis. Foundations and Trends in Information Retrieval, 2(12):1-135.

Sara Rosenthal, Alan Ritter, Preslav Nakov, and Veselin Stoyanov. 2014. Semeval-2014 task 9: Sentiment analysis in Twitter. In Proceedings of the 8th International Workshop on Semantic Evaluation (SemEval 2014), pages 73-80, Ireland.

Richard Socher, Alex Perelygin, Jean Wu, Jason Chuang, Christopher D. Manning, Andrew Y. Ng, and Christopher Potts. 2013. Recursive deep models for semantic compositionality over a sentiment treebank. In Proceedings of the 2013 Conference on Empirical Methods in Natural Language Processing, pages 16311642, USA.

Duyu Tang, Furu Wei, Bing Qin, Ting Liu, and Ming Zhou. 2014. Coooolll: A deep learning system for Twitter sentiment classification. In Proceedings of the 8th International Workshop on Semantic Evaluation (SemEval 2014), pages 208-212, Ireland. 